\title{
Optimized Handoff Decision Mechanisms for Scalable Network Mobility Support*
}

\author{
Sangwook Kang, Yunkuk Kim, Woojin Park, Jaejoon Jo, and Sunshin An \\ Dept. of Electronics \& computer Eng., Korea University, \\ 1, 5-Ga, Anam-dong Sungbuk-ku, Seoul, Korea, Post Code: 136-701 \\ \{klogic, dbs1225, progress, jjj, sunshin\}@dsys.korea.ac.kr
}

\begin{abstract}
Network Mobility (NEMO) is concerned with managing the mobility of an entire network and included one or more Mobile Routers (MRs) which are connected as gateways to the Internet. This paper proposes the optimal handoff decision mechanisms, not only avoiding the 'ping-pong effect' and frequent handoffs, but also reducing handoff latency under multi-hop network mobility. The simulation results demonstrate that the proposed method is well adapted for supporting network mobility over traditional handoff decision algorithms.
\end{abstract}

\section{Introduction}

Compared to approach like Mobile IPv6 (MIPv6) [1] where each host has the mobility support, NEtwork MObility (NEMO) [2, 3, 4] is concerned with managing the mobility of an entire network, with a varying point of attachment to the Internet. This type of network topology is referred to as a mobile network (NEMO) and includes one or more Mobile Routers (MRs) which are connected as gateways to the Internet. The typical examples of a mobile network are PANs (Personal Area Networks), networks of sensors deployed in vehicles, and access networks deployed in public transportation to provide Internet access to devices carried by their passengers. The Internet Engineering Task Force (IETF) NEMO W/G [5] is developing a solution based on MIPv6 with minimal extensions for this mobile network.

In this paper we consider the situation where several mobile networks are deployed in close vicinity and MRs also tend to change their direction of movement very rapidly (e.g. vehicles). In [5], when a MR leaves a network and enters another network, it should perform the handoff operations like MIPv6. Generally a handoff can be decided by the movement detection algorithms $[6,7,8]$, such as Lazy Cell Switching (LCS) and Eager Cell Switching (ECS). The first algorithm, LCS, is based upon the lifetime of the advertisement sent by the router. If a Mobile Node (MN) fails to receive another advertisement from its current network within the specified lifetime, MN should assume it has moved out of range from that network. On the other hand, a handoff in ECS is initiated as soon as a new network is discovered. That is, when a

\footnotetext{
* This research was supported by the MIC (Ministry of Information and Communication), Korea, under the ITRC (Information Technology Research Center) support program supervised by the IITA (Institute of Information Technology Assessment).
} 
MN detects an advertisement with a different network identifier than the current network, MN assumes that a handoff has happened. These approaches may seem simple and effective to decide a handoff. However, these approaches are unsuitable in NEMO environments where a MR is moving rapidly with a random direction and many MRs are deployed in close vicinity. The first approach can result in a problem called the "ping-pong effect", and the second method may result in considerable handoff latency. Thus, a new handoff decision algorithm is necessary to support optimal handoff operation in multi-NEMO environments.

Furthermore, if a MR which has a wireless transceiver is located more than one hop away from the Access Router (AR), i.e., outside of the propagation scope of AR, MR cannot access directly to the AR and should use its neighbor MRs to access the Internet. But, when MR is located within range of two or more neighbors (ARs or MRs) and is receiving advertisements from all of them, MR cannot distinguish which router is its parent, i.e., the upstream router that destined to the Internet [9]. That is to say, when a new advertisement is received from neighbors, MR cannot decide whether sender is a fixed AR that directly connected to the Internet or a MR which attached to the AR or an isolated MR. So when a moving MR receives the RA from an isolated MR earlier than the other MR which connected to the Internet, a moving MR may think it is under a fixed network and forward its outgoing packets to that MR. In this situation if a moving MR wants to send the packets to the outside of mobile network, the packets cannot be routed correctly. Accordingly, in order to provide uninterrupted services and continuous communication in such NEMO environments, we must consider mechanisms for efficient handoff support.

To solve these problems described in above, this paper proposes the mechanisms for optimal handoff decision under multi-hop NEMO environments, not only supporting Internet connectivity and reducing the routing overhead, but also avoiding the 'ping-pong effect' and frequent handoffs.

The remainder of this paper is organized as follows. In next section, we describe an extended MIPv6's RA message to discover its default router which has the Internet connectivity and then proposes an optimal NEMO handoff procedure. We also propose a new handoff decision algorithm for NEMO. Section 3 evaluates the proposed NEMO handoff decision algorithms. Finally, section 4 concludes this paper.

\section{Optimized Handoff Decision Mechanisms}

\subsection{Extended Router Advertisement (RA) Message}

This section describes an extended MIPv6's RA message to discover its default router which has the Internet connectivity and support network mobility under multi-hop NEMO environments. Extending the Prefix Information option of the RA message is suggested as follows:

First, an extra flag ' $\mathrm{H}$ ' taken from the 'reserved 1' field is used to distinguish the type of sending router when a MR receives advertisements from neighbors. If this flag is unset, it means that a sender is not a fixed AR but a MR operating away from home. If a normal RA message, as defined in [10], is received, the sender is a fixed 
AR connected to the Internet. Accordingly, a moving MR should advertise an extended RA with the ' $\mathrm{H}$ ' flag periodically to neighbors until it returns back to its home network.

Second, a new ' $\mathrm{D}$ ' flag is used to decide whether a sending MR has information regarding connectivity to the Internet. If an advertisement from the new neighbor is received and the ' $D$ ' flag containing in this message is unset, it indicates that the sending MR does not currently have information about its default router, i.e., the sender is an isolated MR. If this flag is set, it means that sending router has information about the Internet connectivity and the 'Network Prefix' field is included a delegate Care-of-Address (DCoA) to access the Internet. In here we use the AR's address as the DCoA for all MNNs within the NEMOs. In this case the network prefix of the received RA message is computed by the leftmost "Prefix Length" bits of sender's address.

Finally, the 'Network Level (NLevel)' field taken from the 'reserved 2' field is defined as the number of hop between a MR and AR, which is used to establish parentchild relationships between MRs. This field is initialized to one by AR's child-MR, i.e. MR that attached to an AR directly, and its value is increased using the distance from AR.

\subsection{Optimal NEMO Handoff Procedure}

In this section, the procedure to decide an optimal handoff under multi-hop NEMO environments is described. As illustrated in Fig. 1, an optimal handoff decision can be partitioned into four phases as follows:

\section{Phase I : Check the reachability of the default router}

In this phase, MR checks the reachability of the current default router and counts the number of RA missed of default router. MR relies on RA message to know whether it is still attached to its default router. For reachability confirmation, MRs keep a counter that counts the number of RAs missed for its default router.

MRs can assume they have missed at least one advertisement if the RA interval passes without receiving an advertisement from its default router, so MRs increase RA miss counter. If the consecutive missing RAs reach three times, the MR decides that it loses reachability with its default router. In this case a MR must attempt registration with a known neighbor or solicit for the discovery of other neighbors. The number of RAs missed (represented as ' $\mathrm{N}$ ') is used to calculate the Internet Connectivity Strength (ICS) of its default router in Phase III.

\section{Phase II : Neighbor router's type decision}

As explained earlier, a moving MR advertises an extended RA periodically to neighbors until it returns back to its home network. If MR receives a normal RA message, as defined in [10], the sender is a fixed AR connected to the Internet. On the other hand, if an extended RA is received and " $\mathrm{D}$ " flag contained in this message is set, the sender which sent this message is a MR which had already attached to the Internet. If the ' $\mathrm{D}$ ' flag contained in an extended RA is unset, the sender is an isolated MR.

Once the decision of sender's type is completed, the process of ICS calculation for handoff decision is initiated as illustrated in Fig. 1. 


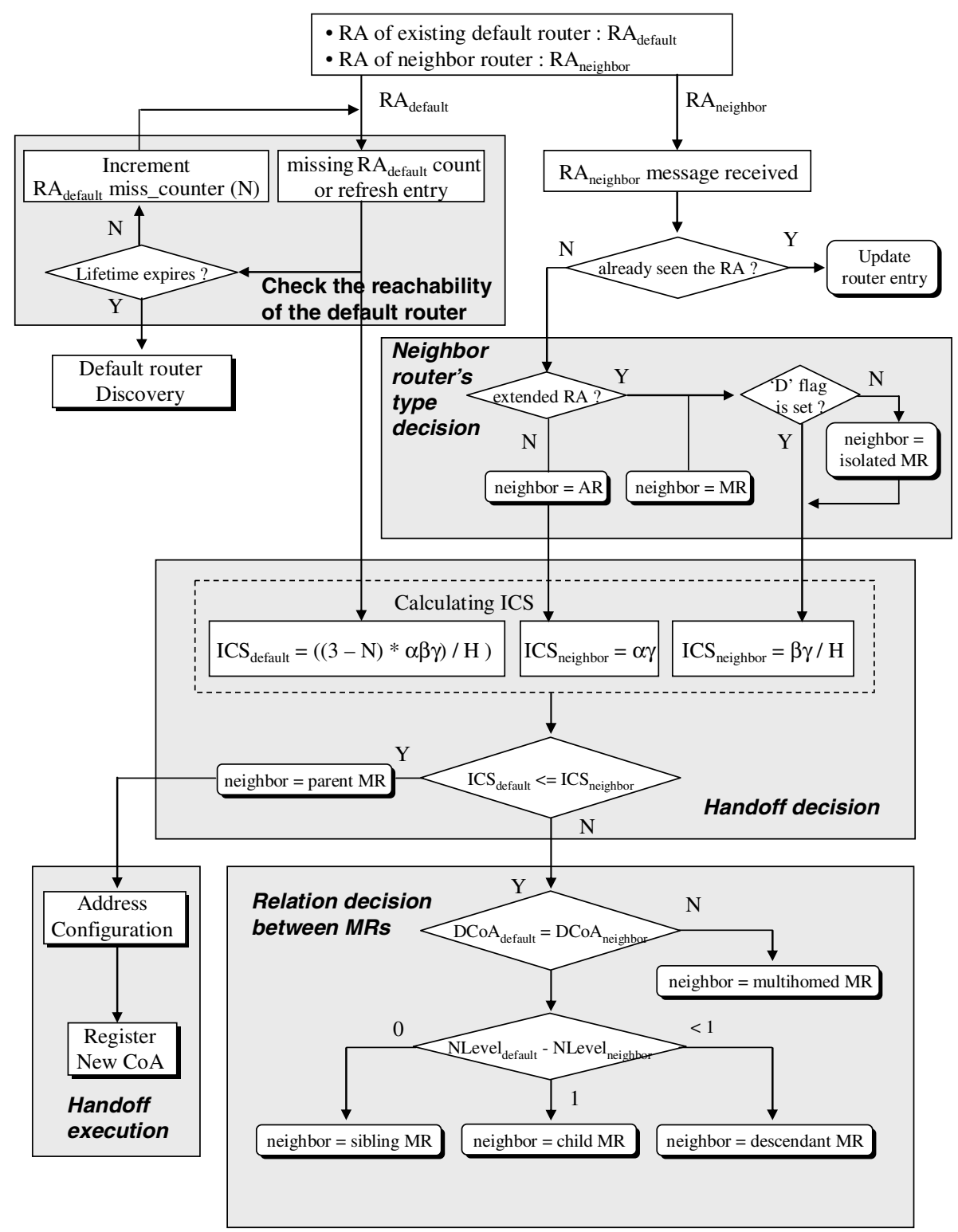

Fig. 1. Decision tree for optimized NEMO handoff

\section{Phase III : Handoff decision and execution}

In this phase, MR calculates the ICS of its default and neighbor router, respectively, and then determines whether it performs handoff decision process based on the values of ICS calculated in previous phase. If the ICS of the neighbor is greater than that of its default router, a handoff will be performed. If handoff execution is determined, 
MR performs handoff process, such as address configuration and register new CoA. Otherwise, MR will proceed with the phase of relation decision between MRs. The handoff decision algorithm for NEMO is discussed in detail in the section 2.3.

\section{Phase IV : Relation decision between MRs}

This phase describes the process of relation establishment between MRs. In our proposal, each MR can know both the DCoA, i.e., AR's address to access the Internet, and its network level thanks to information included in an extended RA message. Accordingly, every time the MR receives the RAs sent by new MRs in its coverage area, it performs the operation of relation establishment between MRs as following:

1) If $\mathrm{DCoA}_{\text {neighbor }}=\mathrm{DCoA}_{\text {default }}$ and $\mathrm{NLevel}_{\text {neighbor }}=\mathrm{NLevel}_{\text {default }}+1$, a sending $\mathrm{MR}$ becomes its child MR. In this case a MR stores information about child mobile network in its routing table.

2) If $\mathrm{DCoA}_{\text {neighbor }}=\mathrm{DCoA}_{\text {default }}$ and $\mathrm{NLevel}_{\text {neighbor }}>\mathrm{NLevel}_{\text {default }}+1$, a sending $\mathrm{MR}$ becomes its descendant MR. In this case a MR ignores this RA message because a parent MR has shortest hop distance to a AR than that of the descendant MR.

3) If $\mathrm{DCoA}_{\text {neighbor }}=\mathrm{DCoA}_{\text {default }}$ and $\mathrm{NLevel}_{\text {neighbor }}=\mathrm{NLevel}_{\text {default }}$, a sending MR becomes its sibling MR. In this case a MR stores information about sibling mobile network in routing table as an alternate upstream MR that destined to the AR.

4) If $\mathrm{DCoA}_{\text {neighbor }} \neq \mathrm{DCoA}_{\text {default }}$, this RA message has been transmitted from a MR that has information about AR in different NEMO domain. In this case MR is multihomed. In the NEMO terminology [2], the NEMO is considered multihomed when either the NEMO is simultaneously connected to the Internet via more than one MR, or when a MR has more than one egress interface. In here we make the assumption that the NEMO has only one AR to access the Internet and is not multihomed.

Through this relation establishment, MR places the route entries in its routing table based upon the information gathered in each of the RA message received. In this way, the MR dynamically learns routes to the neighbor MRs in the NEMO domain.

\subsection{Handoff Decision Algorithm for NEMO}

In this section, we propose a new handoff decision algorithm called NEMO Cell Switching (NCS) to support an optimal handoff decision in multi-hop MR environments. The NCS is based on certain handoff criteria called "Internet Connectivity Strength (ICS)" and is contained the advantages of the LCS and ECS (that is, avoiding the 'ping-pong' effect and reducing the handoff delay).

In NCS algorithm, whenever a MR receives the RA messages sent by neighbors before its default router's lifetime expires, it compares the sending router and its current default router by basing on the Strength of the Internet Connectivity (ICS) to decide which is more suitable as its new default router. ICS is defined as

$$
\text { Internet Connectivity Strength (ICS) }=\alpha \beta \gamma / \mathrm{H}
$$

- $\alpha$ (Type of sending router) : If sending router is a fixed AR, a MR can access directly to the Internet. On the contrary, if sending router is a MR, it means that $\mathrm{MR}$ is located more than one hop away from the AR. In this case MR cannot 
access directly to the AR and should use its neighbor MRs to access the Internet as ad-hoc mobile networking. From this viewpoint one may say that ICS of AR is greater than that of the MR. In our proposal, the type of sending router is determined by the form of received RA message, that is, either normal RA [10] or extended RA message.

- $\beta$ (State of Internet connectivity) : This parameter is used to determine whether sending router can access to the Internet. In our proposal, if a AR's advertisement is received or ' $\mathrm{D}$ ' flag contained in the extended RA is set, one value is always assigned to the sender. If ' $D$ ' flag is unset, i.e. sending router is an isolated $\mathrm{MR}$, zero value is assigned to the sender.

- $\gamma$ (Domain similarity) : This parameter is used to decide handoff types, such as intra/inter domain handoff. In here 'Domain' is defined as set of all MR via the same AR to access the Internet, i.e. tree topology. After receiving RA messages, MR always checks the 'D' flag and 'Network Prefix' field contained in this message. If the 'D' flag is set and sender's DCoA matches its default router's one, the MR learns that it is still moving within the same tree domain, and needs to handle local mobility. Otherwise, the MR learns that it has entered a new tree domain, and would handle inter-domain handoff. Compared to inter-domain handoff, intra-domain handoff can reduce both handoff latency and signaling load by eliminating registration between MR and remote HA. Thus, Internet connectivity in intra-domain handoff is greater than that of inter-domain handoff.

In proportion as the parameter $\alpha, \beta$ and $\gamma$ mentioned in the above rise the ICS increases. On the other hand, the ' $H$ ' parameter indicates the hop distance between sending router (or default router) and AR. Due to mobility of MRs, the topology of connection from sending router (or its default router) to AR may be quite dynamic. If the hop distance toward AR is long, that ICS can not be stable. Hence, a parameter ' $\mathrm{H}$ ' is defined in inverse proportion to rises of ICS.

During handoff, packets addressed to MR may be lost. Packet loss will be significant, especially when the handoff process occurs frequently. This problem will degrade the communication performance. To avoid unnecessary handoff, such as 'ping-pong' problem, priority is given to a default router as described in Equation (2).

$$
\mathrm{ICS}_{\text {default }}=((\text { RA's Lifetime }) * \alpha \beta \gamma) / \mathrm{H}=(3-\mathrm{N}) * \alpha \beta \gamma / \mathrm{H}
$$

where the RA's lifetime is set at three times of the interval and ' $N$ ' is the number of RAs missed.

Generally MR can fail to receive a RA message from its current default router. In our proposal MR keeps a counter that counts the number of RAs missed for its default router. The count is incremented on the expiry of RA interval. If the missing RA count reaches doubles, ICS of the current default router is the same as Equation (1). In the case of three consecutive missing RAs, MR concludes that its current default router as unreachable because the $\mathrm{ICS}_{\text {default }}=$ zero. This is same as LCS algorithm.

An Equation (1) also can define as shown in the below according to the type of sending router.

$$
\begin{aligned}
& \mathrm{ICS}_{\text {sender }}=\alpha \gamma, \text { if sending router is a fixed AR or } \\
& \mathrm{ICS}_{\text {sender }}=\beta \gamma / \mathrm{H}, \text { if sending router is a MR or }
\end{aligned}
$$




$$
\mathrm{ICS}_{\text {sender }}=\text { zero }, \text { if sending router is an isolated MR }
$$

As described in the above, after receiving a new RA message, MR calculates the ICS of its default router and sending router, respectively. If the ICS of the sending router is greater than that of its default router as Equation (3), a handoff will be performed.

$$
\mathrm{ICS}_{\text {default }}<\mathrm{ICS}_{\text {sender }} ; \text { handoff execution }
$$

\section{Performance Evaluation}

To show how well NCS algorithm performs, we compare the performance of the three handoff decision algorithms, i.e. ECS, LCS and NCS. The simulation was based on the Network Simulator (NS-2) [11] and Mobiwan [12] developed by the Motorola. The scenario we have studied includes 32 mobile networks that are placed randomly over a rectangular $(800 \mathrm{~m} \times 800 \mathrm{~m})$ flat space for 1,000 seconds of simulated time, and are connected to the ARs with a hierarchical tree structure, i.e., tree-based NEMO scheme. To simulate real traffic, we set up the $\mathrm{CN}$ as a traffic source of a Constant Bit Rate (CBR) source over a User Datagram Protocol (UDP), producing fixed length packets of 1000 bytes each every 1 second. A moving MR1 acts as a sink receiving packets from $\mathrm{CN}$.

Table 1. Parameters for NCS Algorithm

\begin{tabular}{c|l|l}
\hline \hline Parameter & \multicolumn{1}{|c}{ Description } & Values used in simulation \\
\hline $\mathrm{N}$ & Number of RAs missed & $0 \sim 3$ \\
$\mathrm{H}$ & $\begin{array}{l}\text { Hop count from its default router (or a } \\
\text { sending router) to AR }\end{array}$ & $1 \sim$ \\
$\alpha$ & Type of sender (or its default) router & 2 (AR) \\
& & 1 (MR) \\
$\beta$ & State of Internet connectivity & 0 (AR or 'D' flag is set) \\
$\gamma$ & Domain similarity & 2 (same domain) \\
& & 1 (different domain) \\
\hline \hline
\end{tabular}

Since the handoff decision algorithm concerns the communication between the AR (or MR) and MRs, we have only focused on the wireless part of the scenario. In order to simulate handoff decision efficiently, mobility speed of a moving MR1's neighbors (MR2 MR32) set to zero, i.e., have kept fixed with $0 \mathrm{~m} / \mathrm{s}$ during all simulations, and propagation delay is ignored. The RAs period for these MRs is 1 second, but the RAs from these MRs are not synchronized.

When comparing the performance of the handoff decision algorithms, we use two metrics, i.e. handoff frequency and handoff frequency, in terms of the mobility speed, the number of MRs and RA intervals, respectively. In this simulation, the main parameters used for NCS are shown in Table 1.

\section{Mobility Speed and Handoff Performance}

Fig. 2 shows simulation results for handoff performance when the mobility speed of a moving MR is varied. In Fig.2 (a), ECS has higher handoff frequency than other two 


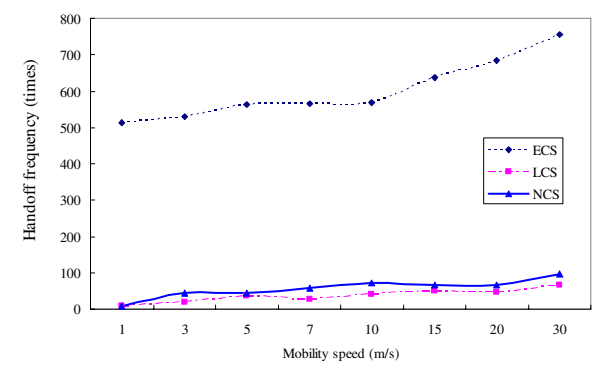

(a) Handoff frequency

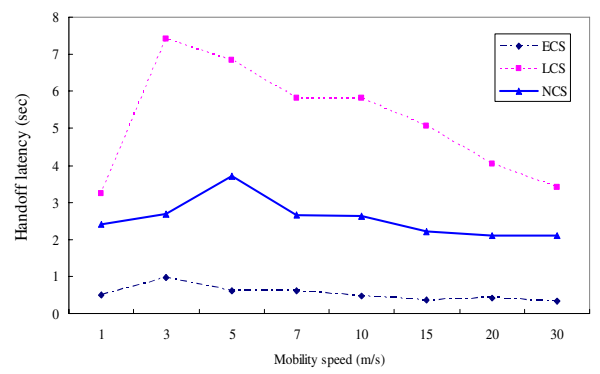

(b) Handoff latency

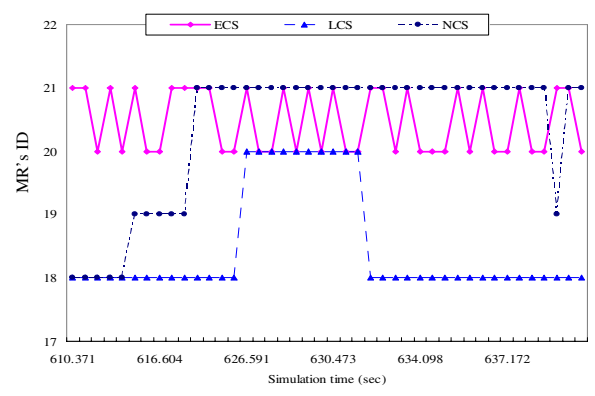

(c) Ping-pong handoffs

Fig. 2. Mobility Speed and Handoff Performance

algorithms. It is believed that a handoff initiates immediately upon learning a new network prefix. Unlike ECS, LCS and NCS perform well though the frequency increases slightly as the mobility speed increases. This is because MR does not initiate a handoff until the current point of attachment is confirmed to be unreachable (LCS) or until the ICS of a new neighbor is greater than that of its current router (NCS).

The handoff latency of three algorithms shows in Fig. 2 (b). In Fig. 2(b), the handoff latencies associated with LCS and NCS are high than that of ECS. This is because it takes some time to determine whether to perform a handoff (NCS) or to wait until its current router is confirmed to be unreachable (LCS). However, compared to the LCS, the NCS offers lower handoff latency. It is because MR in NCS initiates a handoff whenever the Internet connectivity of a new neighbor is greater than that of its current router. From the results, the handoff latency of ECS is decreased slightly for the speed of the MR. This is because that the possibility of receiving a new RA increases as the mobility speed increases, and a handoff in ECS initiates immediately upon receiving a new RA.

In Fig. 2(b), ECS may offer better handoff latency performance than both LCS and NCS, but it will result in unnecessary handoff to the other neighbor MR as shown in Fig. 2 (c). We see that in the case of ECS a handoff is performed repeatedly between MR20 and MR21. That is, the ping-pong problem happens frequently in ECS, unlike LCS and NCS. 


\section{Number of MRs and Handoff Performance}

Fig. 3 is to evaluate the performance of three handoff decision algorithms as a function of the number of MRs, which is located within NEMO domain. In Fig.3 (a), Both LCS and NCS exhibit the lower handoff occurrence, i.e. the handoff frequency is slightly increased as the number of MRs increases. This is because that the decision whether or not to perform a handoff is decided by a reactive handoff initiation method. That is, a moving MR does not initiate a handoff until the current network becomes unavailable (LCS) or until the ICS toward a new network is greater that of its current network (NCS). Therefore, we can see that handoff frequency of both LCS and NCS does not influenced greatly by the number of MRs. On the contrary, as the number of MRs increase, the handoff frequency of ECS increases rapidly. This is due to two main reasons:

- ECS is to change network as soon as a new network is discovered. Thus, the possibility of handoff occurrence is high in NEMO domain where many MRs are deployed.

- If many MRs are deployed in close vicinity, a moving MR can be located in wireless environments with two or more overlapping RAs. This will result in the so called "ping-pong" effect which makes the MR switches between the neighbors within coverage.

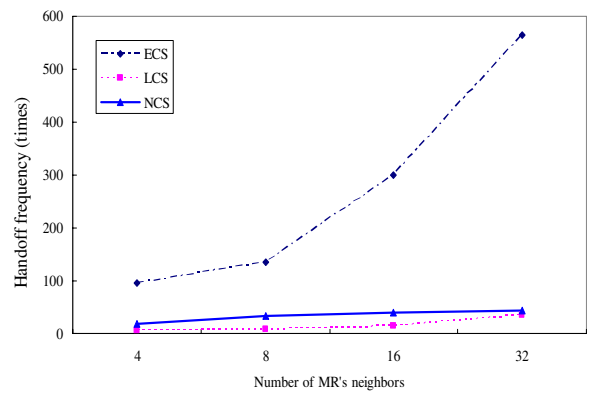

(a) Handoff frequency

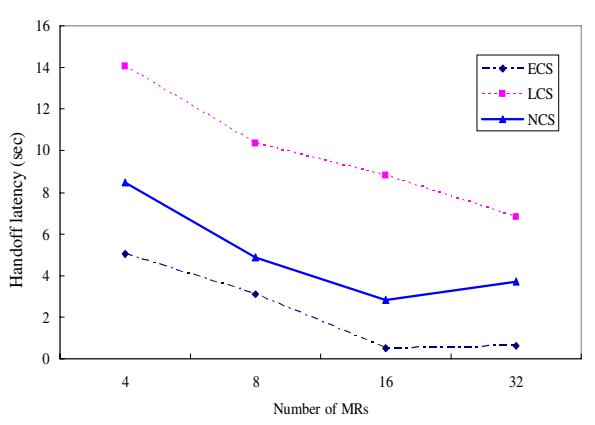

(b) Handoff latency

Fig. 3. Handoff frequency vs. Number of MRs

Fig. 3 (b) illustrates the handoff latency. On the whole, as the number of MRs increase, the latency decreases. This is because that it takes long time to find a new default router at the small number of MRs after/before the current router's lifetime is expired. When the number of MR goes over 16, the latency begins to vary constantly. As expected, since LCS is never initiated before the current network is declared unreachable, handoff latency of LCS is high than that of both ECS and NCS. Compared ECS, NCS handoff latency is high slightly since NCS evaluates a set of handoff criteria and determines which MR provides the best performance from a moving MR point of view.

\section{RA Interval and Handoff Performance}

As NEMO uses the reception of RAs to discover new networks, the interval between sending RAs can affect the time it takes to discover new networks. Generally, frequent 
RA is helpful for movement detection and it causes shorter handoff latency [13, 14]. However, processing frequent RA is significant overhead for MRs in the view of networks.

Fig. 4 shows the handoff performance of a moving MR when the interval of the MR's RA is varied. As expected, when the interval between RAs is 0.5 seconds in Fig. 4 (a), ECS exhibits the higher handoff occurrence. This is because that fast RA interval can directly affect the behavior of ECS and a moving MR performs unnecessary handoff, i.e. ping-pong, continuously at overlapped zone between the neighbors. When the interval of RAs goes over a certain value, the handoff frequency begins to decrease rapidly. In both LCS and NCS, handoff occurs constantly between 0.5 seconds and 2 seconds. This is because that both LCS and NCS handoff initiation methods are not influenced by the interval between receiving unsolicited RAs and by the reachabilty current network.

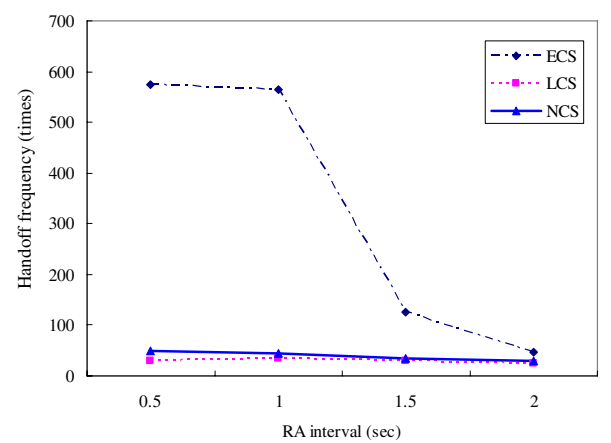

(a) Handoff frequency

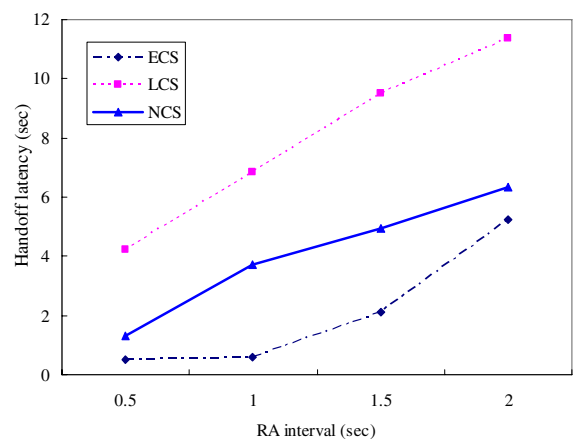

(b) Handoff latency

Fig. 4. RA intervals vs. Handoff performance

Fig. 4 (b) shows the average of handoff latency when the interval of RA is varied. The simulation results say that all the handoff decision methods are increased rapidly as the interval of RAs increases. This is because that the time of handoff detection which triggers a handoff to a new network increases according to the interval increasing. Compared to the ECS, LCS and NCS offer lower handoff latency.

\section{Conclusion}

In this paper, the mechanisms for optimal handoff decision under multi-hop NEMO environments, not only supporting Internet connectivity and reducing the routing overhead, but also avoiding the 'ping-pong effect' and frequent handoffs, are proposed.

First, extending the MIPv6's RA message is suggested to discover its default MR which has the Internet connectivity, and we then described the optimized handoff decision procedure to decide an optimal handoff under multi-hop NEMO environments. This procedure is partitioned into four phases: 1) Check the reachability, 2) Neighbor router's type decision, 3) Handoff decision and execution, and 4) Relation 
decision between MRs. Next, a new handoff decision algorithm called NEMO Cell Switching (NCS) is proposed to support an optimal handoff decision in multi-hop MR environments. The NCS is based on certain handoff criteria called "Internet Connectivity Strength (ICS)".

To evaluate the performance of the handoff decision mechanisms proposed, we have performed a series of simulations. The simulation was based on the Network Simulator (NS-2) and MobiWAN developed by Motorola. The simulations have been executed under various simulation environments taking into account mobility speed, the number of mobile networks, RA intervals. The simulation results show that the proposed approach, i.e., NCS algorithms, outperforms the existing approaches in most cases. Thus, we believe that the work presented is an important step towards supporting optimal handoff.

\section{Reference}

[1] D. Johnson, C. Perkins, J. Arkko, "Mobility Support in IPv6", RFC 3775, June 2004

[2] Thierry Ernst, Hong-Yon Lach, "Network Mobility Support Terminology", < draft-ietfnemo-terminology-01.txt>, Feb. 2005

[3] V. Devarapalli, R. Wakikawa and P. Thubert, "Nemo Basic Support Protocol”, RFC 3963, Jan. 2005

[4] T.Ernst,, Keisuke Uehara and Koshiro Mitsuya "Network Mobility from the InternetCAR perspective", Advanced Information Networking and Applications, 2003. AINA 2003. 17th International Conference, pp. 19 - 25, March 2003

[5] IETF NEMO WG, http : //www.mobilenetworks.org/nemo

[6] N.Blefari-Melazzi, M.Femminelle, F.Pugini, "Movement detection in IP heterogeneous wireless networks", Personal Mobile Communications Conference, 2003. 5th European (Conf. Publ. No. 492), pp. 121 - 125, April 2003

[7] N. Blefari-Melazzi, M. Femminella, F. Pugini, "A robust and flexible solution for Movement Detection in Wireless IP Networks: Enhanced Lazy Cell Switching", Proceedings of IST Mobile \& Wireless Telecommunications Summit 2002, June 2002

[8] Daley, G., Pentland, B., Nelson. R, "Movement detection optimizations in mobile IPv6", ICON2003, The 11th IEEE International Conference, pp. 687 - 692, Sept. 2003

[9] H. Cho, E. K. Paik, "Hierarchical Mobile Router Advertisement for nested mobile networks", < draft-cho-nemo-hmra-00.txt>, work in progress, Jan. 2004

[10] T. Narten, E. Nordmark and W. Simpson, "Neighbor Discovery for IP Version 6 (IPv6)", RFC 2461, Dec. 2004

[11] The VINT Project, The UCB/LBNL/VINT Network Simulator-ns (version2), http:// www.isi.edu/nsnam/ns.

[12] MobiWAN: NS-2 extensions to study mobility in Wide-Area IPv6 Networks, http://www.inrialpes.fr/planete/pub/mobiwan/

[13] Daley G., Pentland B., Nelson R., "Movement detection optimizations in mobile IPv6", Networks, 2003. ICON2003. The 11th IEEE International Conference, pp. 687 - 692, Sept. 2003

[14] Daley G., Pentland B., Nelson R., "Effects of fast routers advertisement on mobile IPv6 handovers", Computers and Communication, 2003. (ISCC 2003), Eighth IEEE International Symposium, pp. 557 - 562, 2003 